\title{
Multi-scale Salient Feature Extraction on Mesh Models
}

\author{
Yong-Liang Yang ${ }^{1,2}$ and Chao-Hui Shen ${ }^{2}$ \\ 1 King Abdullah University of Science and Technology, Thuwal, KSA \\ 2 Tsinghua University, Beijing, China
}

\begin{abstract}
We present a new method of extracting multi-scale salien$\mathrm{t}$ features on meshes. It is based on robust estimation of curvature on multiple scales. The coincidence between salient feature and the scale of interest can be established straightforwardly, where detailed feature appears on small scale and feature with more global shape information shows up on large scale. We demonstrate this multi-scale description of features accords with human perception and can be further used for several applications as feature classification and viewpoint selection. Experiments exhibit that our method as a multi-scale analysis tool is very helpful for studying $3 \mathrm{D}$ shapes.
\end{abstract}

\section{Introduction}

Due to the fast development of 3D scanning and modeling technology, triangular meshes are now widely used in computer graphics. Objects with fruitful surface details can be well captured and constructed into mesh form. The interests in analyzing the geometric information of meshes are ever increasing. This is the most important step for a variety of applications in computer graphics, computer vision and geometric modeling, such as shape retrieval, shape alignment, feature preserved simplification etc.

In shape analysis, the key is how to define intrinsic features which can well represent the model's characteristic. To ensure the intrinsic property, the features are often required to be invariant under rigid transformation and uniform scaling. Moreover, the extracted feature should be discriminative to other models especially with different type. Based on different feature definition, shape analysis method can be generally classified into two categories: global and local [1]. The former one focuses on describing the entire shape of the model with a so-called "shape descriptor". The methodology of 3D statistics like shape distribution and histogram are usually involved, while local geometric details are not concerned much. On the other hand, local method defines features based on local surface properties. Curvature and its related quantities are often used here.

There have been several publications about determining saliency or extracting salient features on meshes in the recent years. [2] defined a measure of mesh saliency using a center-surround operator on Gaussian-weighted mean curvatures. This work incorporates insights from human perception, while the extraction of interesting feature parts is not their concern. [3] defined salient feature as 


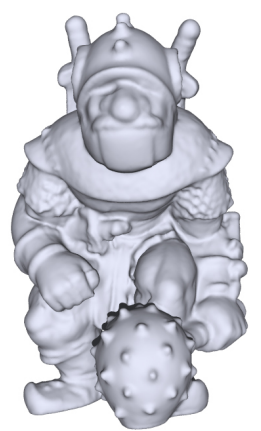

(a)

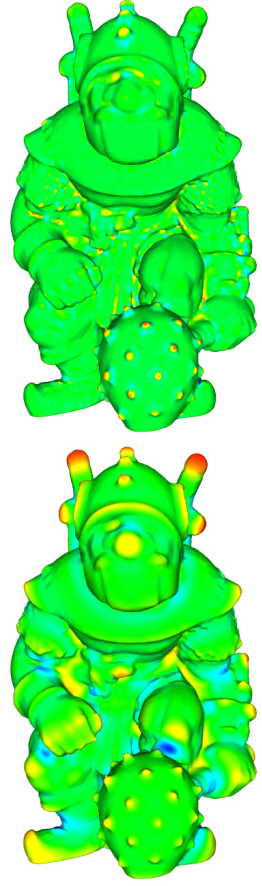

(b)

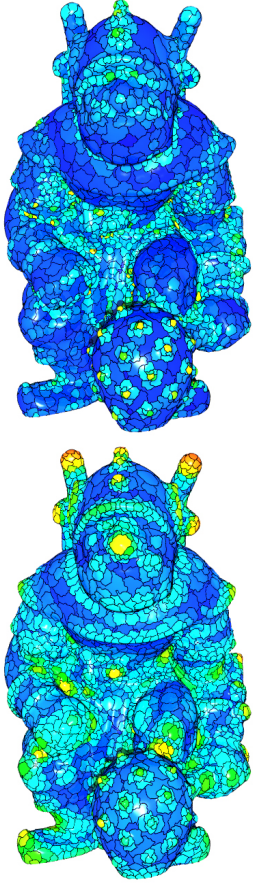

(c)

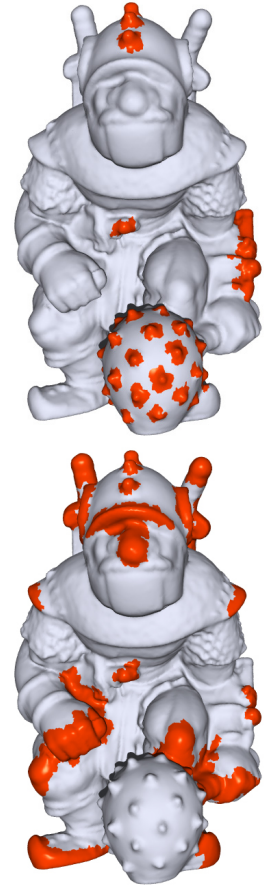

(d)

Fig. 1. Multi-scale salient feature extraction. (a) Grog model; (b) Gaussian curvature on small and large scales (from top to bottom, similarly hereinafter); (c) Local surface descriptors on small and large scales; (d) Salient features extracted accordingly.

region with high importance and non-trivial local shapes. They proposed to extract salient features based on curvature from local fitting, but there is no scale specialty of features considered here. Shilane et al. [4] presented a novel method to select regions that distinguish a shape by not only judging the shape itself. It is based on performing a shape-based search using each region as a query into a database. This method can reasonably select the regions which successfully discriminate the model with others, but the precondition is the availability of a shape retrieval environment. Recently, Chen et al. [5] investigated the so-called 'schelling points' on 3D surface. These points have to be manually selected by the users beforehand on a training data set. Then features can be predicted on new shapes based on the prior knowledge.

In this paper, we present a method of extracting salient geometric features on multiple scales. It is more likely to analyze local shape properties, while global shape information is taken into account when the scale of interest becomes large (see Fig. 1). Although the definition of salient feature is also based on curvature and its variance, the curvature estimation is performed in a multi- 
scale way. The salient features extracted on different scales represent different level of surface details. We show that the scale specialty of salient features can help us to understand the surface shape more comprehensively.

The rest of the paper is organized as follows: In Section 2, we will describe the procedure of multi-scale salient feature extraction in detail. Two interesting applications which benefit from our method will be presented in Section 3. Finally, we conclude our paper in Section 4 and discuss some of the future work.

\section{Multi-scale salient feature extraction}

In this section, we present our multi-scale salient feature extraction algorithm in detail. For the geometric meaning of salient feature, we adapt to use the definition in [3], where salient feature is defined as compound high-level feature of non-trivial local shapes. Compared with the features represented per mesh vertex (cf. [2]), it conveys much more shape information of the local geometry. In their definition, the criterion of the salient local shape is related to its saliency and interestingness which is determined by curvature and its variance. However, the curvature information they used is from local fitting and no scale specialty is taken into account. In our paper, we propose to extract the salient geometric feature based on curvature estimated on different scales. In this way, we can further judge the feature property whether it belongs to the surface detail or it represents surface more globally.

\subsection{Multi-scale curvature estimation}

Instead of computing curvature based on local quadric fitting, we use multi-scale curvature estimation in [6]. The principal curvatures and the principal frame are estimated by principal component analysis $(P C A)$ of local neighborhoods defined via spherical kernels centered on the given surface. The neighborhood radius $r$ can be naturally treated as the scale of interest. In this paper, we use $P C A$ of the ball neighborhood for multi-scale curvature estimation for all examples. Fig. 2 shows the maximal principal curvature of the Asian Dragon model estimated on two different scales. Note that the scale features are more apparently recognized on the small scale.

\subsection{Local surface descriptor generation}

Based on the multi-scale curvature information which has been successfully estimated, a sparse set of local surface descriptors $(L S D)$ will be built across the mesh surface afterwards. Each $L S D$ is a surface point $p$ and its associated quadric patch that approximate the surface in a local neighborhood of $p[3]$. This kind of $L S D$ has many advantages: adaptive to the geometry of the shape, independent of the underlying triangulation, heavily reduce the complexity of the original triangle mesh representation and ease of clustering non-trivial salient features. 

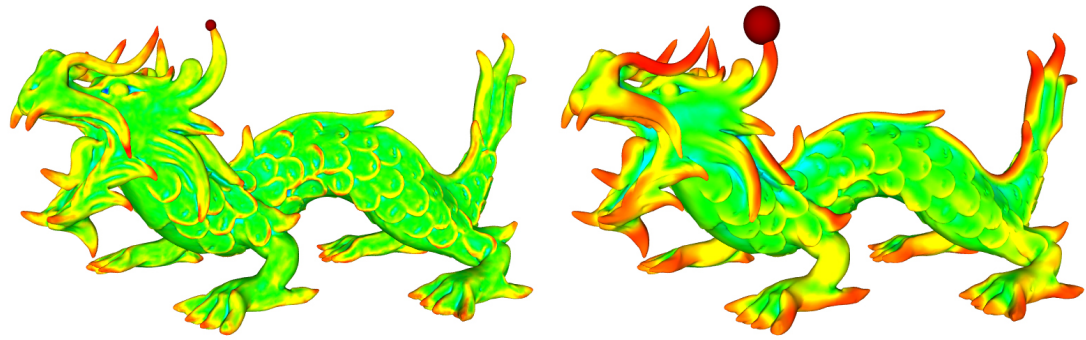

Fig. 2. Multi-scale maximal principal curvatures on the Asian Dragon model, with two different kernels centered at one of its horn. The red color depicts the highest curvature value, blue color is for lowest value.(upper: small scale; bottom: large scale.)

In [3], the $L S D$ of a surface point $p$ is built based on the geometric error between the local surface patch and the fitting quadric. However, in our method, the curvatures are estimated using PCA of local neighborhoods. In this case, the small shape variance can be neutralized on a large scale (see Fig. 2), which means the geometry itself can not reflect the change of the scale. So instead of using vertex coordinates, we build the $L S D$ based on the curvature information, which is correlated with the scale of interest.

We also use the region-growing technique to iteratively generate the $L S D$ s. First, we sort all the mesh vertices according to their curvature function value $\operatorname{Curv}(p)$ in descending order. The curvature function can be chosen depending on the model's property. Commonly we choose the absolute Gaussian curvature, and for CAD models, the maximal absolute principal curvature will be used (see [3]). Then we build the $L S D$ s one by one from the sorted list. For a vertex $p$ in the list which hasn't been in any $L S D$, we extract its associated quadratic patch in a way different from local fitting.

As discussed in Section 2.1, based on $P C A$ of local neighborhood of a surface point $p$, we get three eigenvectors which form its local principal frame on scale $r$ besides principal curvatures $\kappa_{1}$ and $\kappa_{2}$. Then we form the paraboloid $P: z=$ $\frac{1}{2}\left(\kappa_{1} x^{2}+\kappa_{2} y^{2}\right)$ in principal frame as the second order approximation of the surface at $p$ on the given scale. To generate the $L S D$ from $p$, we greedily involve its neighbor vertices and integrate the error of Gaussian curvature over the local area until the prescribed threshold is reached. Suppose $q$ is one of its neighbor, we can get the local coordinates of $q$ by projecting it into $p$ 's principal frame. Then we only use the local $\mathbf{x}, \mathbf{y}$ coordinates $q_{x}$ and $q_{y}$ to compute the Gaussian curvature $\hat{K}_{G}^{q}$ of the local osculating paraboloid as in Equ. (1).

$$
\hat{K}_{G}^{q}=\frac{\kappa_{1} \kappa_{2}}{\left[1+\left(\kappa_{1} q_{x}\right)^{2}+\left(\kappa_{2} q_{y}\right)^{2}\right]^{2}}
$$

The error of the Gaussian curvature can then be estimated as the difference between $\hat{K}_{G}^{q}$ and $K_{G}^{q}=\kappa_{1}^{q} \cdot \kappa_{2}^{q}$, where $\kappa_{1}^{q}$ and $\kappa_{2}^{q}$ are the principal curvatures of $q$ estimated in Section 2.1. Note that in this way, we don't involve local 


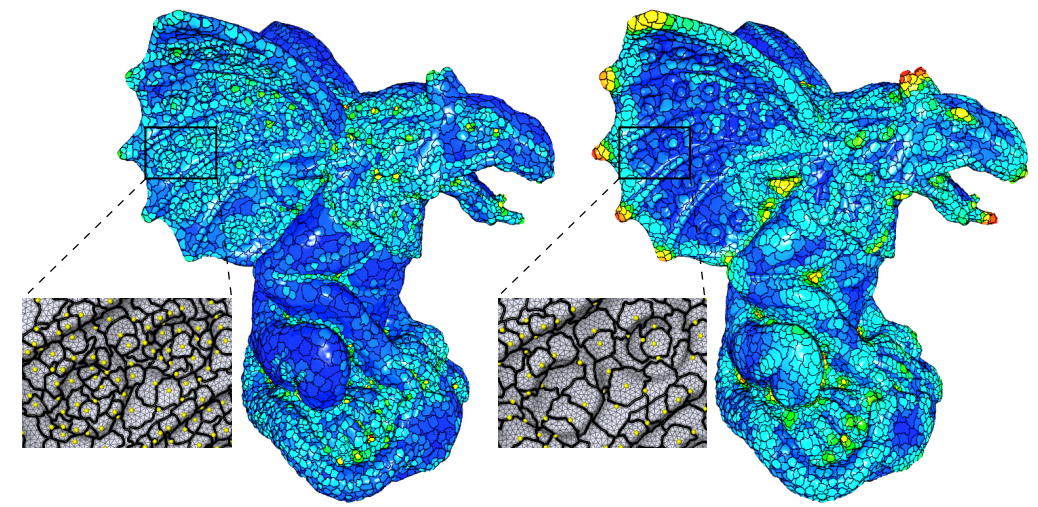

Fig. 3. Local surface descriptors of Gargoyle model on two different scales. Red is high curvature function value and blue is low. Zoomed figures show the tiny structure and starting point of each LSD. (left: small scale; right: large scale.)

z coordinate, this can eliminate the error of $L S D$ caused by the local shape variance when the scale becomes large.

In our implementation, for the model less than $100 \mathrm{~K}$ faces, we use 0.3 of its largest absolute Gaussian curvature times the average area per-vertex as the threshold. For large models, we set the ratio to 1.0. After a single $L S D$ with starting point $p$ is extracted, we assign it with the largest curvature function value, i.e. $\operatorname{Curv}(p)$, as the representative curvature value.

Fig. 3 shows the local surface descriptors of Gargoyle model on two different scales. We can find the $L S D$ s on small scale follow the surface detail (the ringlike shape) better, while descriptors representing global curved shape are salient on large scale (see also zoom-in parts).

\subsection{Salient feature extraction}

The definition of saliency or salient feature is the foundation of distinctiveness analysis of 3D shapes. Due to its generality and our purpose of extracting multiscale salient feature regions, we adapt to use the definition and measurement of salient feature in [3]. They define salient feature as a cluster of $L S D$ s that locally describe a non-trivial region of the surface.

For each $L S D$, we grow a cluster of descriptors by recursively adding its neighboring descriptors until the saliency grade of the clustered feature is maximized. This greedy process stops when the contribution of a candidate descriptor is insignificant. The saliency grade of a feature cluster is determined by the curvature function value of each $L S D$ and its variance over the cluster. We refer readers to [3] for the details.

When the whole surface has been decomposed into feature clusters, the ones with high saliency grade will be extracted as salient geometric features. This can 


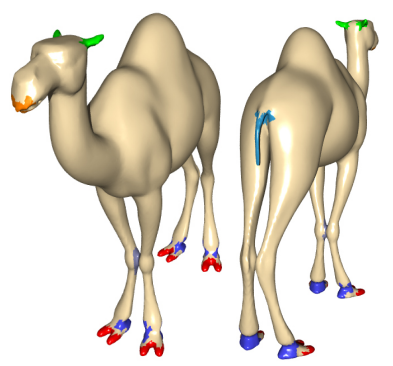

(a)

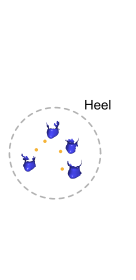

(b)

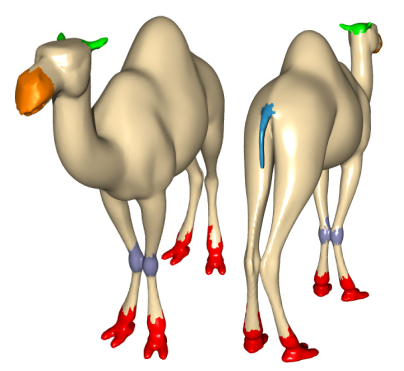

(c)

Fig. 4. Feature classification of camel model based on salient feature extraction on two different scales. (a) the classified salient features with different colors on small scale; (b) the 2D projection of the salient feature space computed using classical multidimensional scaling; (c) the classified salient features on large scale.

be done by a prescribed threshold of the saliency grade value or the percentage of salient features among all clusters. Since concave feature is usually generated by adjacent meaningful convex parts [7], we suppress its saliency grade so that the inherent salient feature can be successfully extracted. The results of salient feature extraction of Grog model on two different scales can be found in Fig. 1.

\section{Results and discussion}

\subsection{Multi-scale feature classification.}

Our feature classification is based on multi-scale salient features in Section 2 . The goal is to classify salient features on different scales according to their global shapes, i.e. the salient features which have similar shape will be grouped into the same class. We believe this way of multi-scale salient feature extraction and classification will give us a comprehensive understanding of 3D models.

In our method, we use spin-image [8] as the shape signature for each salient feature extracted from a 3D model. The resemblance between salient features is measured by their spin-images. A full distance matrix is generated afterwards. Then we extract a $2 \mathrm{D}$ embedding of the salient feature space using multidimensional scaling [9]. From the feature space, we obtain a meaningful classification of salient features.

Fig. 4(a) shows the salient feature classification results of Camel model on small scale. We can see the meaningful body parts like ears, toes, heels, mouth, tail and joint of front legs are successfully classified as in Fig. 4(b). The classification results on large scale of the same Camel model can be found in Fig. 4(c). The salient features capture more global interesting shape of the surface. Toes and heels are merged to whole foot features. 

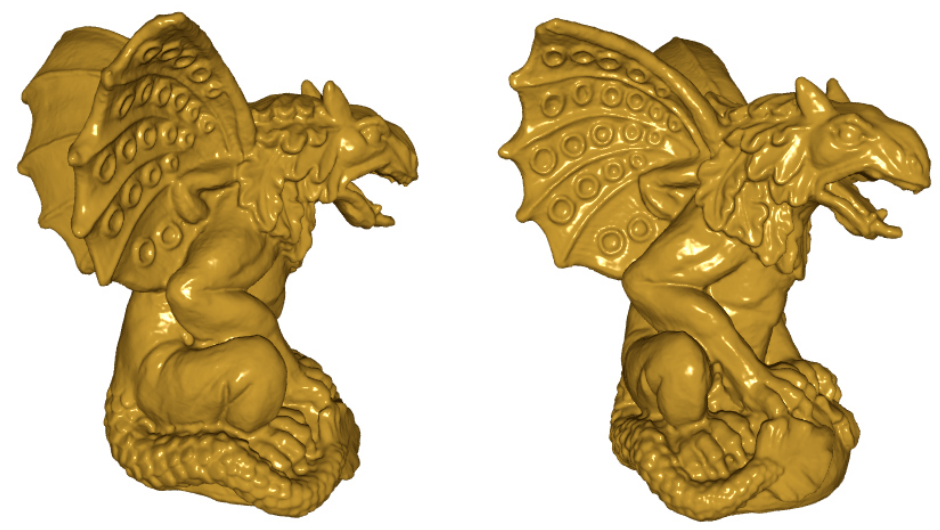

Fig. 5. Multi-scale viewpoints selected on two different scales for Gargoyle model (left: large scale; right: small scale).

\subsection{Multi-scale viewpoint selection.}

Based on our multi-scale salient feature extraction, we can do the viewpoint selection on multiple scales. In our approach, different viewpoint is determined by visible surface saliency on different scales. The visual effect is like observing an object from far to near. On large scale, features with some global shape information show up, while more details of an object are revealed on small scale. The intuition behind our approach is that people tend to notice global shape features of an object at first and then pay attention to more detailed ones. Thus, our approach helps to provide an informative illustration of a 3D object, with global and detailed features visible on different scales.

In our method, we define the saliency of a mesh vertex $v$ as $S(F) / \operatorname{Size}(F)$, where $F$ is the salient feature which contains vertex $v, S(F)$ is the saliency grade of $F, \operatorname{Size}(F)$ is the number of vertices that belong to $F$. For vertex which doesn't belong to any salient feature, the saliency value is 0 . After that, we search for the viewpoint which maximizes the sum of saliency of all visible vertices. To avoid the sharp variance of saliency between neighboring viewpoint, here we set top $50 \%$ feature clusters as salient features.

Fig. 5 shows two optimal salient viewpoints of the Gargoyle model, which are selected on large and small scale respectively. Note that the two wings of Gargoyle model are more visible on large scale, while the detailed features (e.g. the rings) are more attractive on small scale.

\subsection{Implementation details}

Our multi-scale salient feature extraction algorithm is implemented in $\mathrm{C}++$ on a windows platform. In all our experiments, we scaled the models to fit into 
a bounding box with maximal length 2 . On small scale, the radius of the ball neighborhood is set to 0.03 and on large scale it is $3 \sim 4$ times larger.

We test our algorithm on an Intel Core2 Duo $2.66 \mathrm{GHz}$ computer with $2 \mathrm{~GB}$ RAM. For Camel model with $70 \mathrm{~K}$ triangles, the average cost of salient feature extraction on a single scale is $38.9 \mathrm{~s}$. For Grog model with 200k triangle, the cost is $40.5 \mathrm{~s}$. The curvature estimation step takes most of the time. On the other hand, the extraction of local surface descriptors and salient features are much more efficient due to the greedy approach, these two processes can be done within 5 seconds for all test models.

\section{Conclusion and future work}

In this paper, we presented a new method of multi-scale salient feature extraction. The salient features extracted on small scale represent the surface detail while more global interesting salient regions can be extracted on large scale. This kind of multi-scale description of features accords with human perception from different scales of interest. We also applied the multi-scale salient feature extraction to feature classification and viewpoint selection, both applications show that our method as a multi-scale analysis tool is very helpful for studying 3D shapes.

We want to apply the multi-scale salient feature extraction to a wider usage like in shape matching, where different models can be compared on different scales. Models have details in common have more similarity on small scale while models with similar global features are expected to be matched on large scale. We believe this kind of multi-scale feature based shape matching is favorable of further applications like modeling by example and shape retrieval.

\section{References}

1. Tangelder, J.W., Veltkamp, R.C.: A survey of content based 3d shape retrieval methods. smi 00 (2004) 145-156

2. Lee, C.H., Varshney, A., Jacobs, D.W.: Mesh saliency. ACM Trans. Graph. 24(3) (2005) 659-666

3. Gal, R., Cohen-Or, D.: Salient geometric features for partial shape matching and similarity. ACM Trans. Graph. 25(1) (2006) 130-150

4. Shilane, P., Funkhouser, T.: Distinctive regions of 3d surfaces. ACM Trans. Graph. 26(2) (2007) 7

5. Chen, X., Saparov, A., Pang, B., Funkhouser, T.: Schelling points on 3D surface meshes. ACM Trans. Graph. 31(3) (2012)

6. Yang, Y.L., Lai, Y.K., Hu, S.M., Pottmann, H.: Robust principal curvatures on multiple scales. In: Symposium of Geometry Processing. (2006) 223-226

7. Katz, S., Tal, A.: Hierarchical mesh decomposition using fuzzy clustering and cuts. ACM Trans. Graph. 22(3) (2003) 954-961

8. Johnson, A.: Spin-Images: A Representation for 3-D Surface Matching. PhD thesis, Robotics Institute, Carnegie Mellon University, Pittsburgh, PA (August 1997)

9. Cox, T., Cox, M.: Multidimensional Scaling. Chapman \& Hall, London (2001) 